\title{
A relação entre renda e nível educacional e a experiência dos trabalhadores nos diferentes estratos de rendimentos no Norte do Brasil
}

\section{The relationship between income and educational level and the work experience considering the different income strata in the North Region of Brasil}

Luan Oliveira Queiroz - Mestrando em Desenvolvimento Econômico e Políticas Públicas da Universidade Federal de Viçosa. E-mail: luanqoliveira@gmail.com

Francisco Carlos da Cunha Cassuce - Doutor em Economia Aplicada pela Universidade Federal de Viçosa. Professor adjunto do Departamento de Economia da Universidade Federal de Viçosa. E-mail: francisco.cassuce@ufv.br

Jader Fernandes Cirino - Doutor em Economia Aplicada pela Universidade Federal de Viçosa. Professor adjunto do Departamento de Economia da Universidade Federal de Viçosa. E-mail: jader.cirino@ufv.br

\section{Resumo}

A ideia é analisar os efeitos da educação e da experiência dos indivíduos sobre seus ganhos salariais nos diferentes estratos de rendimento da região Norte do Brasil. A metodologia adotada para o estudo utiliza o Modelo Econométrico de Regressão Quantílica com base nas informações disponibilizadas da PNAD 2013, onde se retirou os dados sobre os rendimentos e as variáveis de caracterização socioeconômicas destes indivíduos. Os resultados indicaram que a experiência do trabalhador e a sua escolaridade relacionamse positivamente com o rendimento destes trabalhadores, com a ressalva da relação não linear entre a experiência e a renda. A análise dos impactos da educação e da experiência sobre a renda nos distintos estratos mostrou que os retornos são maiores à medida que os indivíduos apresentam maiores rendimentos. Enquanto que a análise dos efeitos marginais apontou que concluir o ensino médio tem maior impacto sobre o ganho daqueles que obtêm menores rendimentos.

\section{Palavras-chave}

Nível Educacional. Experiência no Trabalho. Rendimento. Região Norte do Brasil. Regressão Quantílica.

\begin{abstract}
This article aims to analyze the effects of education and experience on individuals wage gains considering the different income strata in the North region of Brazil. To this, the method of Quantile Regression was used. The information about incomes and other socioeconomic variables were extracted from the National Household Survey 2013. The results show that the worker's experience and schooling have a positive influence over his income, although the relation found between experience and income was not linear. The impact analysis in the different strata showed that returns of education and experience over income are higher in the superior quantiles. Also, the marginal effects analysis showed that obtaining high school degree has a higher impact on the lower levels of income.
\end{abstract}

\section{Keywords}

Schooling Level. Work Experience. Income. North Region of Brazil. Quantile Regression. 


\section{INTRODUÇÃO}

Muitos estudos buscam explicar ou relacionar as heterogeneidades salariais e a produtividade do trabalho dos indivíduos. Becker (1964), Mincer (1974) e Langoni (1973), no Brasil, são os trabalhos seminais que primeiro idealizam a relação entre salário e produtividade, utilizando como variáveis explicativas o nível de escolaridade (proxy da produtividade) e a experiência. Com a ressalva de que a teoria econômica supõe que trabalhadores mais qualificados geram mais produtos ou bens. Estes trabalhos são importantíssimos, dada à compreensão de que as desigualdades salariais, a priori, surgem de atributos ou características econômicas (como, por exemplo, nível de aprendizado dos trabalhadores) daqueles inseridos no mercado de trabalho.

No entanto, alguns artigos avaliaram essas questões para o Brasil, e a percepção é de que essas desigualdades são geradas também por fatores não econômicos e não observados como, por exemplo, mulheres que geralmente apresentam níveis mais elevados de educação, em média, se comparadas aos homens, recebem menos em termos salariais, considerado um mesmo posto de trabalho (ARAUJO; RIBEIRO, 2002).

Becker (1962) compreende que os investimentos em capital humano (teoria do capital humano), dada a análise do custo-benefício que os indivíduos fazem entre investir em aprimoramento das habilidades técnicas ou entrar no mercado de trabalho, produzem efeitos sobre determinada dimensão da renda decorridos da força de trabalho. Ou seja, de forma agregada, a educação é vista como um investimento no estoque de recursos humanos. E o nível de escolaridade e a qualidade da educação passam a ser consideradas peças-chave para a discussão de desenvolvimento econômico.

Langoni (1973) traz essa discussão para o cenário nacional, adicionando a estas variáveis as características gênero, setor de atividade e região de residência. E a partir deste estudo e as críticas ao modelo econométrico estimado, vários modelos econométricos surgem para relacionar educação e renda, no intuito de melhor explicar a situação de crescimento e desenvolvimento econômico brasileiro.

Mincer (1974) acredita que os ganhos salariais estão intimamente ligados com o nível de escolaridade. No entanto, deve-se ter cautela para avaliar os modelos com base em educação, sendo de extrema relevância a incorporação da experiência no mercado do indivíduo para analisar os ganhos, uma vez que essas variáveis indicam a noção de "learning by doing" e de mobilidade do trabalho, que também influenciam na variação dos rendimentos. 
Uma gama de estudos contemplam a relação entre o grau de escolaridade e experiência do trabalhador e o nível de renda foi realizada para o Brasil, o que será discutido na revisão da literatura. Porém, poucos trabalhos preocuparam-se em analisar estas relações e as suas possíveis dinâmicas no contexto regional, no caso deste artigo, a Região Norte do Brasil. O objetivo é de buscar características bem delineadas desta região que possam aprofundar o entendimento sobre a dinâmica deste mercado de trabalho, com ênfase na relação analisada.

E quando se almeja observar a dinâmica do mercado de trabalho de uma região, é conveniente ressaltar a possibilidade de que estas relações entre educação e renda possam ser atenuadas ou intensificadas à medida que se altera o nível de renda dos trabalhadores, sobretudo num mercado de trabalho considerado em formação e desenvolvimento, como é no caso do Norte do Brasil. Deste modo, há necessidade de se especificar em quais estratos de rendimento estes ganhos salariais decorrentes do aumento do nível educacional ou da experiência adquirida dos trabalhadores ficam mais evidentes.

É interessante observar que na Região Norte, como em qualquer outra, uma das carências é a geração de empregos e oportunidades que possibilitem suprir as demandas sociais da população. E este tipo de análise favorece e enriquece a discussão sobre o desenvolvimento econômico regional adequado para tal, e pode fornecer informações para a formulação de políticas públicas e quais os estratos e perfis de trabalhadores as diretrizes dos programas voltados para a geração e qualificação do emprego devem abranger quando se analisa a região.

Diante destes desdobramentos surgem algumas questões: Em que medida o nível de formação e experiência dos trabalhadores poderia impactar na remuneração destes profissionais no mercado de trabalho? Existem outras variáveis que possam expressar ou explicar essas heterogeneidade dos rendimentos para esta região? A relação analisada é distinta nos diferentes estratos de renda desses trabalhadores para este mercado de trabalho?

Neste sentido, o objetivo deste estudo é identificar os estratos de rendimento no Norte do Brasil, onde a relação entre renda, nível educacional e experiência dos trabalhadores é mais significativa; e quais os atributos econômicos mais importantes quando observadas estas diferenças, na perspectiva de produzir estudos capazes de entender as várias dinâmicas do mercado de trabalho da região. Os objetivos específicos são: (i) Verificar se as diferenças salariais são significativas para as diferentes características econômicas; (ii) Identificar em quais estratos de renda essas desigualdades são mais significativas; (iii) Buscar possíveis respostas na literatura contemporânea que expliquem essas diferenças para a região. 


\section{REVISÃO DE LITERATURA}

A teoria do capital humano de Becker (1964) afirma que os salários são diferentes, devido à relação entre salário e produtividade ou atributos econômicos (como experiência no trabalho), sem que haja qualquer tipo discriminação. Para estabelecer essa relação, considera escolaridade como uma variável proxy da produtividade. Isto significa que os investimentos dos indivíduos em educação produzirão efeitos nas suas habilidades logo, na sua produtividade marginal, visando obter ganhos em seus rendimentos, gerados por estes esforços em busca da sua qualificação (BECKER, 1964; MINCER, 1974).

Essa teoria considera a idade como proxy da experiência no trabalho, que, neste caso, capta a habilidade e a inteligência dos indivíduos num processo denominado pela teoria econômica de "learning by doing," ou seja, das habilidades e inteligências individuais, que surgem a partir das práticas laborais e do treinamento no trabalho (BECKER, 1964; MINCER, 1974). Além de observar a relação econômica entre experiência e mobilidade do trabalho, em que os indivíduos adquirem mais experiência por estar em constante rotação em diversas atividades, o que pode representar o desenvolvimento de habilidades e técnicas diferenciais - de certa forma advinda do treinamento no trabalho nesta gama de ocupações ao longo da trajetória profissional - que facilita sua inserção no mercado de trabalho e numa variação de rendimentos, dada a sua capacidade técnica (BECKER, 1964; MINCER, 1974).

No entanto, deve-se levar em conta dois aspectos sobre esta pressuposição teórica. Em primeiro lugar, conforme Cirino e Cassuce (2012), a diferença entre nível de escolaridade e a qualidade deste aprendizado, o que levaria, caso não fizesse esta distinção, à superestimação dos resultados. Em segundo lugar, o efeito parábola, que consiste em observar uma relação positiva entre ganho salarial e experiência no mercado de trabalho até certo nível (próximo à idade média do indivíduo), quando há uma, depreciação do capital humano, dada a condição física dos trabalhadores, que tende a deteriorar ao longo do tempo (BECKER, 1964; MINCER, 1974; BERNDT, 1996). Para contemplar este último efeito, recomenda-se utilizar, além da variável idade, esta na forma quadrática (MINCER, 1974).

Desta forma, Mincer (1974), visando comprovar que os retornos na renda dos indivíduos estão diretamente ligados ao nível de formação e experiência no trabalho, estabelece um modelo econométrico no intuito de demonstrar esta relação na realidade. Esta verificação ficou conhecida como função-salário do capital humano ou equação minceriana, e matematicamente pode ser expressa pela equação: 
[...] em que é o logaritmo natural do salário ou do rendimento do trabalho do indivíduo; S, é a escolaridade do trabalhador medida em anos de estudo; J, é a experiência do indivíduo contabilizada através dos seus anos no mercado de trabalho; $(\mathrm{k}=0$ a 3) são os parâmetros a serem estimados; e é o termo de erro estocástico com as propriedades usuais (MINCER, 1974).

A relação presumida teoricamente sobre a equação (1) é que existe uma relação positiva entre a escolaridade a experiência do trabalhodor com o rendimento advindo do seu trabalho, ou seja, por suposição, os coeficientes são positivos e, portanto, maiores que zero. No entanto, considerando que o incremento na experiência deste trabalhador estaria sujeito a retornos decrescentes (depreciação do capital humano), implica que o coeficiente é negativo, o que torna a função parabólica, côncava em J.

A partir destas considerações, elencará os principais estudos sobre a temática proposta e o que deve ser ressaltado neste artigo. O primeiro trabalho que deve ser mencionado é o de Langoni (1973), que consistiu em criar um modelo econométrico, com aporte na Teoria do Capital Humano, regredindo a renda sobre o nível de escolaridade, a idade (experiência no trabalho), o sexo (sob a hipótese de que mulheres são menos produtivas que homens), setor de atividade econômica e região da residência do indivíduo (LANGONI, 1973). Langoni (1973, p. 116) considera que a desigualdade salarial está "intimamente associada à própria estrutura qualitativa da força de trabalho", representada pelas cinco variáveis explicativas do modelo.

Kassouf (1994) introduz a correção de seletividade amostral via procedimento de Heckman nos estudos nacionais, na tentativa de estimar equações de participação no mercado de trabalho e determinação de salários para mulheres e homens de 16 a 71 anos de idade, uma vez que a estimação por meio de MQO pode produzir indicadores tendenciosos. Este autor considera que as estimativas de salários podem ser usadas como custo de oportunidade do tempo dos trabalhadores (KASSOUF, 1994). Uma de suas considerações é que quando utilizado o método tradicional, observou-se uma tendenciosidade positiva nas estimativas dos parâmetros para o caso dos homens e uma tendenciosidade negativa para trabalhadores do sexo feminino (KASSOUF, 1994).

Sachsida, Loureiro e Mendonça (2004), por meio de um modelo econométrico baseado na equação minceriana, investigam o retorno obtido na renda pela escolaridade para o Brasil, utilizando MQO (Mínimos Quadrados Ordinários) e corrigindo os possíveis vieses de seleção por diferentes procedimentos - de Heckman (1979) e o de Garen (1984). Uma das principais 
considerações do trabalho é acerca dos retornos em escolaridade tenderem a variar conforme o nível de escolaridade do trabalhador e a existência de endogeneidade na escolha da escolaridade, advinda da procura por emprego (SACHSIDA; LOUREIRO; MENDONÇA, 2004).

Outro trabalho importante é o de Matos e Machado (2006), que consiste na avaliação dos diferenciais de rendimentos, observando a existência ou não de discriminação por sexo e cor. O método utilizado foi a decomposição OaxacaBinder, onde é possível analisar de forma mais detalhada o diferencial de renda com base nas variáveis de interesse (MATOS; MACHADO, 2006). As variáveis escolhidas para construir o modelo são: cor, gênero, idade, grande região de residência, ramo de atividade, relação na família, posição na ocupação, anos de escolaridade e rendimento real por hora (MATOS; MACHADO, 2006). E tal decomposição foi realizada sobre a equação minceriana, com base nas variáveis explicativas indicadas anteriormente. Os resultados mostraram que no período entre 1987 a 2001, os diferenciais no rendimento tinham como parcela explicada a discriminação por sexo e cor, ou seja, umas parcelas da heterogeneidade salarial advinha de atributos não econômicos (MATOS; MACHADO, 2006).

Rezende e Wyllie (2006) buscam medir econometricamente os retornos para educação no Brasil, com referência aos dados da pesquisa sobre Padrão de Vida (PPV-IBGE). Os autores consideram na sua análise uma equação minceriana incluindo variáveis dummy de intercepto para raça, região, tipo da área (metropolitana ou rural) e posição no domicílio (REZENDE; WYLLIE, 2006). Além de estimarem equações de rendimentos para homens e mulheres, utilizam o procedimento para correção do efeito de seletividade amostral de Heckman e incluem uma medida de qualidade para educação (REZENDE; WYLLIE, 2006).

Arraes e Mariano (2014), por meio de equações mincerianas, buscam identificar e mitigar os efeitos da endogeneidade da variável educação sobre as distintas taxas de retorno da educação. Além de considerar diferentes modelos para estimar estas taxas, os autores desconsideram a endogeneidade e adotam o uso de variáveis instrumentais e tratamento teórico adequado à equação minceriana, por meio de modificações na relação entre capital humano e regionalização, no intuito de escolherem o melhor modelo preditivo (ARRAES; MARIANO, 2014). Segundo estes autores os diferenciais das estimativas da taxa de retorno do capital humano podem estar ligados à qualidade dos instrumentos disponíveis.

Por fim, Dalberto (2014) analisa a diferença de rendimento entre trabalhadores informais e formais da Região Metropolitana de Belo Horizonte. Para isto utilizam a decomposição Oaxaca-Blinder quantílica de Melly (2006). As variáveis para estimação do modelo baseiam em equações mincerianas 
e são calculadas por meio de Regressão Quantílica. São elas: o logaritmo dos rendimentos do trabalho, a escolaridade do trabalhador (segmentado em quatros grupos), em anos de estudo, a idade do trabalhador como proxy da experiência, a cor do trabalhador e o setor de atividade (DALBERTO, 2014). Os autores perceberam indícios da presença de segmentação no mercado de trabalho da $\mathrm{RMBH}$, principalmente relacionados a aspectos sobre a distribuição de rendimentos (DALBERTO, 2014).

Dessa forma, o presente estudo almeja contribuir para a literatura sobre o tema ao investigar o impacto do nível educacional do trabalhador e sua experiência no mercado de trabalho sobre o rendimento do trabalho em nível regional. Para tanto, de acordo com a metodologia adotada, estimaram-se Regressões Quantílicas com base em uma equação minceriana construída a partir das variáveis controles e indicada recomendada pela literatura utilizada.

Destaca-se que é esperado que os efeitos marginais da educação e da experiência sobre a renda nos diferentes estratos são distintos à medida que os indivíduos apresentam rendimentos maiores que a mediana. Ou seja, além do impacto destas variáveis ser positivo nos rendimentos dos trabalhadores, a intensidade sobre a magnitude também varia de acordo com os diferentes quantis da amostra.

\section{METODOLOGIA}

Para atingir os objetivos propostos, a metodologia adotada para o estudo prevê a utilização do Modelo Econométrico de Regressão Quantílica, que capta essas informações de forma estratificada conforme os objetivos estabelecidos. Este método apresenta como vantagens a robustez na presença de outliers na variável resposta e melhor descrição de uma posição central da distribuição condicional da variável resposta, quando a distribuição dos erros não é normal (SANTOS, 2012).

As equações mincerianas deste estudo foram calculadas utilizando o método de regressão quantílica (KOENKER; BASSET, 1978). Empiricamente e considerando as variáveis de interesse, tal modelo estimado para os rendimentos pode ser representado como:

Onde são os parâmetros estimados para o quantil $\theta$ da variável dependente, nesse caso o logaritmo dos rendimentos do trabalho, ; $(k=1, \ldots, 4)$ são variáveis discretas que indicam a escolaridade do trabalhador, em anos de estudo, com classe inicial formada por trabalhadores com 0 a 3 anos de estudo e, respectivamente, por trabalhadores com 4 a 7,8 a 10, 11 e mais de 11 anos de estudo; Ida representa a idade do trabalhador como proxy da experiência; e $\mathrm{Ida}^{2}$ representa o efeito parábola; é uma variável dummy que indica o sexo do trabalhador, assumindo o valor 1 para o trabalhador 
do sexo masculino e 0 se for do sexo feminino; $(m=1,2)$ são variáveis dummies que indicam a cor do trabalhador, com o grupo referência sendo formado por brancos, assumindo o valor 1 para pretos e 0 , caso contrário, e assumindo o valor 1 para pardos e zero, caso contrário; $(k=1, \ldots, 4)$ são variáveis dummies que indicam o setor de atividade do trabalhador, sendo o grupo referência formado pela indústria, representando construção, o comércio, a administração pública e os serviços; é uma variável dummy que indica a localização da residência do trabalhador, assumindo o valor 1 para o trabalhador na cidade e 0 se estiver localizado na zona rural; e representa os erros estocásticos da regressão.

Espera-se que quanto maior o nível de escolaridade, maior a produtividade do indivíduo e maior a renda auferida ao profissional. A mesma análise deve ser feita entre experiência do trabalhador e renda. No entanto, há ressalva de que a depreciação física do trabalhador lhe impõe, a certa idade, rendimento decrescente, o que pode ser captado pela variável idade ao quadrado, ou seja, espera-se uma relação inversa entre esta variável e a renda.

A escolha das variáveis de controle, como gênero, setor de atividade, cor, setor de ocupação e região da residência, deram-se pelo direcionamento dos estudos contemplados na revisão de literatura, que apontaram estas características como bons controles para a relação de escolaridade e experiência e a renda para o Brasil, partindo da hipótese de que fatores não econômicos podem estar intimamente ligados ao nível de renda dos trabalhadores no contexto nacional.

A amostra ${ }^{1}$ utilizada é constituída por trabalhadores entre 18 e 54 anos de idade no mercado de trabalho da região Norte do Brasil. Isto se deve à tentativa de contemplar apenas os indivíduos que alcançaram a maioridade, além da exclusão dos possíveis aposentados. Os indivíduos que compõem a amostra são pessoas que estavam trabalhando no período da pesquisa, o que dispensa o procedimento de Heckman² (1979) de viés de seleção amostral.

As informações utilizadas provêm da PNAD 2013 (Pesquisa Nacional por Amostra de Domicílios) que é um levantamento realizado anualmente pelo IBGE, exceto nos anos de Censo (CIRINO, 2008; IBGE, 2011). Esta pesquisa consiste em uma amostra probabilística de domicílios, realizada em todo o território

Esta amostra é composta por 19.266 informações.

O procedimento de Heckman (1994) consiste na consideração da hipótese que para os indivíduos entrarem no mercado de trabalho, o salário praticado neste deve ser maior do que o salário de reserva, determinado implicitamente ou subjetivamente pelo indivíduo (SACHSIDA; LOUREIRO; MENDONÇA, 2004). Logo, o procedimento consiste na perspectiva de considerar os vários critérios distintos de participação do mercado, para não analisá-los de modo idêntico e evitar o viés de seletividade amostral. Isto é claro quando se considera modelos nos quais consideram a participação ou não de indivíduos no mercado de trabalho, por exemplo, em modelos Probit, que buscam estimar a probabilidade de indivíduos estarem empregados. 
nacional, sendo a população-alvo os domicílios e as pessoas aí residentes, de onde se obterá informações sobre os rendimentos e variáveis de caracterização socioeconômicas (CIRINO, 2008; IBGE, 2011).

Por fim, cabe destacar que o plano amostral da PNAD é considerado uma Plano de Seleção Amostral Complexo por envolver características de amostragem estratificada e conglomerada, sendo estas peculiaridades: (i) Estratificação: a população inicial é dividida em subgrupos (estratos), sendo em cada um deles selecionado uma subamostra; (ii) Conglomeração: representa seleção de amostra em vários estágios; (iii) Probabilidades desiguais de seleção em um ou mais estágios; e (iv) Ajustes dos pesos amostrais com totais populacionais conhecidos (CIRINO, 2008). Portanto, deve-se considerar o conjunto de dados da PNAD como sendo de amostragem complexa, no intuito de produzir estimadores consistentes, eficientes e não viesados, que incorporem esta influência da conglomeração e estratificação (CIRINO, 2008).

\section{RESULTADOS E DISCUSSÃO}

A ideia é analisar os efeitos da educação e da experiência sobre os ganhos salariais dos indivíduos. Inicialmente será feita uma caracterização dos trabalhadores da Região Norte, em seguda a análise dos resultados gerados pela regressão quantílica.

Em sua maioria, observado os dados da Tabela 1, os trabalhadores desta região são homens $(62,33 \%)$, pardos $(66,73 \%)$, que moram na cidade $(80,41 \%)$, e provavelmente vão estar ocupados no setor de serviços $(45,93 \%)$ ou no setor da administração pública $(18,79 \%)$. Em média, os trabalhadores desta região apresentam um rendimento de $\mathrm{R} \$ 1278,06$ por mês, com 9,67 anos de estudo e idade por volta de 35 anos. É interessante notar que apenas 8,98\% desses indivíduos são negros, que $37,67 \%$ são mulheres e que 19,60\% trabalham na zona rural (Tabela 1). São indícios de um mercado de trabalho que ainda está passando por transformações em sua composição.

A pouca expressão de mulheres e negros nesse mercado pode estar intimamente ligado ao processo de ocupação do Norte, que a partir da década de 1970 passa a ser uma fronteira em expansão econômica e de preocupação governamental, onde a implantação da malha tecno-política, a Superintendência de Desenvolvimento da Amazônia (SUDAM) e a economia da borracha, tornam-se as peças-chave nesse processo, atribuindo às cidades um papel logístico essencial, o que tornou atraente a imigração de vários trabalhadores pardos e brancos de outras localidades para essa região (BECKER, 2001). 
Foi identificado que na Administração Pública há o maior número de ocupações relacionadas ao sexo feminino, estoque de emprego formal (FERREIRA; SOUZA; HARB, 2011). Isto pode ser explicado devido à existência de concursos públicos para a ocupação de vagas, e como estes exigem capacidade técnica e grau de escolaridade mais elevado, e sabendo que o nível de instrução das mulheres tende a ser mais alto que os homens, supõem-se, então, que para este setor apresente maior participação feminina (ARAUJO; RIBEIRO, 2002), enquanto que a indústria de transformação e a construção civil são de participação predominante masculina, além de serem os setores que ultimamente mais alocam mão de obra na Região Norte (FERREIRA; SOUZA; HARB, 2011). Isso pode ser justificado pela natureza dessas atividades, que exigem como qualidade a força física (ARAÚJO; RIBEIRO, 2002).

Tabela 1 - Médias, proporções e desvios-padrão dos trabalhadores para a Região Norte do Brasil, 2013.

\begin{tabular}{|c|c|c|}
\hline Variável & Média & Desvio-padrão \\
\hline Renda (R\$/mês) & 1278,06 & 22,5722 \\
\hline Escolaridade (em anos) & 9,67 & 0,0602 \\
\hline \multirow[t]{2}{*}{ Idade (em anos) } & 34,86 & 0,0914 \\
\hline & \multicolumn{2}{|c|}{ Proporção (\%) } \\
\hline E1 (4 a 7 anos) & \multicolumn{2}{|c|}{$13,94 \%$} \\
\hline E2 (8 a 10 anos) & \multicolumn{2}{|c|}{$22,14 \%$} \\
\hline E3 (11 anos) & \multicolumn{2}{|c|}{$38,64 \%$} \\
\hline $\mathrm{E} 4$ (> 11 anos) & \multicolumn{2}{|c|}{$18,30 \%$} \\
\hline Mulheres & \multicolumn{2}{|c|}{$37,67 \%$} \\
\hline Homens & \multicolumn{2}{|c|}{$62,33 \%$} \\
\hline Rural & \multicolumn{2}{|c|}{$19,60 \%$} \\
\hline Urbano & \multicolumn{2}{|c|}{$80,41 \%$} \\
\hline Brancos & \multicolumn{2}{|c|}{$24,28 \%$} \\
\hline Negros & \multicolumn{2}{|c|}{$8,98 \%$} \\
\hline Pardos & \multicolumn{2}{|c|}{$66,73 \%$} \\
\hline Indústria & \multicolumn{2}{|c|}{$11,02 \%$} \\
\hline Construção & \multicolumn{2}{|c|}{$13,48 \%$} \\
\hline Comércio & \multicolumn{2}{|c|}{$10,78 \%$} \\
\hline Adm. pública & \multicolumn{2}{|c|}{$18,79 \%$} \\
\hline Serviços & \multicolumn{2}{|c|}{$45,93 \%$} \\
\hline
\end{tabular}

Fonte: Elaboração própria, com base nos dados da PNAD 2013. 
Na perspectiva de traçar um perfil mais delineado desses trabalhadores foi estabelecida a escolaridade média, segundo a cor, o sexo e a condição do domicílio (Tabela 2). Buscou-se testar se estas diferenças na escolaridade, segundo estas variáveis de interesse, são significativas ou estatisticamente diferentes.

Foi percebido que as mulheres (11,03 anos de estudo) que trabalham são, em média, bem mais escolarizadas que os homens (8,84 anos de estudo). Em relação à cor, os trabalhadores brancos (10,08 anos de estudo), em média, são mais escolarizados que os pardos (9,41 anos de estudo) e os negros (8,71 anos de estudo). E, por fim, segundo a condição dos domicílios, os indivíduos que trabalham na cidade (10,31 anos de estudo) são bem mais escolarizados que os que trabalham na zona rural (7,05 anos de estudo). Estes resultados corroboram com o panorama nacional e demonstra que esta região segue a tendência nacional (MATOS; MACHADO, 2006; ARAUJO; RIBEIRO, 2002).

O teste utilizado para testar se estas diferenças são estatisticamente significativas foi o Teste "t" de Student para média, no qual foi observado para todas estimativas, no nível de 1\%, que estas diferenças são significativas (Tabela 2).

Tabela 2 - Média de anos de escolaridade dos trabalhadores da região Norte do Brasil segundo sexo, cor e condição do domicílio, 2013

\begin{tabular}{|c|c|c|c|c|c|c|}
\hline \multirow{3}{*}{ Variável } & \multicolumn{4}{|c|}{ Sexo } & & \\
\hline & \multicolumn{2}{|c|}{ Homens } & \multicolumn{2}{|c|}{ Mulheres } & & \\
\hline & Média & $\begin{array}{l}\text { Desvio- } \\
\text { padrão }\end{array}$ & Média & $\begin{array}{l}\text { Desvio- } \\
\text { padrão }\end{array}$ & & \\
\hline Escolaridade (em anos) & 8,84 & 0,0679 & 11,03 & 0,0647 & & \\
\hline \multirow{3}{*}{ Variável } & \multicolumn{4}{|c|}{ Condição do Domicílio } & & \\
\hline & \multicolumn{2}{|c|}{ Urbano } & \multicolumn{2}{|c|}{ Rural } & & \\
\hline & Média & $\begin{array}{l}\text { Desvio- } \\
\text { padrão }\end{array}$ & Média & $\begin{array}{l}\text { Desvio- } \\
\text { padrão }\end{array}$ & & \\
\hline Escolaridade (em anos) & 10,31 & 0,0624 & 7,05 & 0,1288 & & \\
\hline \multirow{3}{*}{ Variável } & \multicolumn{6}{|c|}{ Cor } \\
\hline & \multicolumn{2}{|c|}{ Brancos } & \multicolumn{2}{|c|}{ Pardos } & \multicolumn{2}{|c|}{ Negros } \\
\hline & Média & $\begin{array}{l}\text { Desvio- } \\
\text { padrão }\end{array}$ & Média & $\begin{array}{l}\text { Desvio- } \\
\text { padrão }\end{array}$ & Média & $\begin{array}{l}\text { Desvio- } \\
\text { padrão }\end{array}$ \\
\hline Escolaridade (em anos) & 10,8 & 0,0921 & 9,41 & 0,0653 & 8,71 & 0,133685 \\
\hline $\begin{array}{c}\text { Teste de diferenças para } \\
\text { escolaridade }\end{array}$ & \multirow{2}{*}{\multicolumn{2}{|c|}{ Estatística }} & \multirow[t]{2}{*}{$\begin{array}{c}\text { Valor } \\
\text { calculado }\end{array}$} & \multirow[t]{2}{*}{$P>|t|$} & & \\
\hline & & & & & & \\
\hline
\end{tabular}




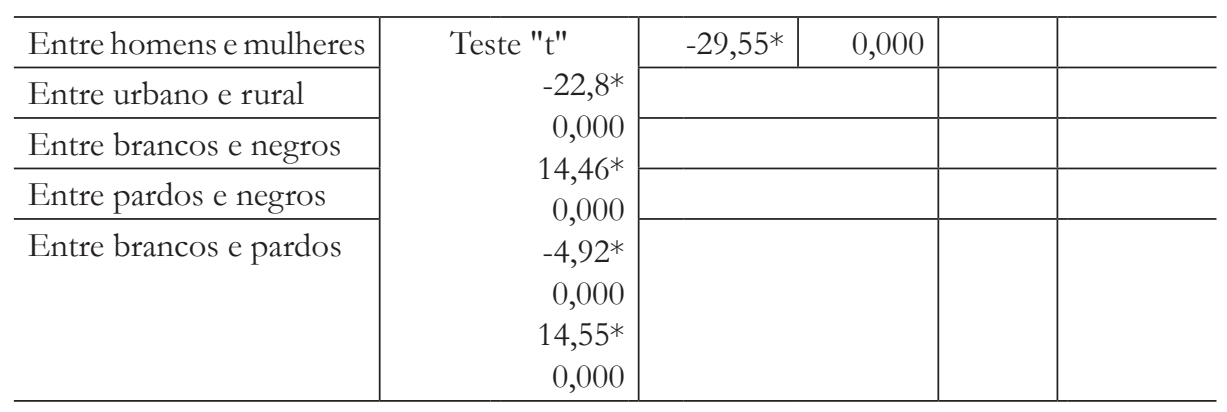

* Significativo a $1 \%$

Fonte: Dados da PNAD 2013.

No intuito de demonstrar que os resultados variam de um quantil para outro, foi calculada a diferença entre os quantis 0,25 e 0,50 e entre os quantis 0,50 e 0,75 , a fim de identifica-se de fato os coeficientes das equações mincerianas de rendimento diferem entre os quantis. Desta forma, foi realizado o teste de igualdade interquantil, que exibe o cálculo dos erros padrão robustos, via Bootstrap, e foca na diferença entre os quantis. Nesse sentido, a regressão interquantil, por meio da utilização do Teste "t" de Student, pode ser entendida como um teste de hipótese sobre as diferenças dos coeficientes de regressão estimados, considerando os respectivos quantis (Tabela 3).

Ao observar a Tabela 3, os coeficientes das variáveis de escolaridade $\left(\mathrm{E}_{3} \mathrm{e}\right.$ $\mathrm{E}_{4}$ ), de cor (negros e pardos) e de setor de atividade (construção) entre os quantis 0,25 e 0,50 e os coeficientes das variáveis que indicam a escolaridade $\left(E_{3}\right.$ e $\left.E_{4}\right)$, a cor (negros), a idade e o sexo entre os quantis 0,50 e 0,75 devem ser considerados estatisticamente diferentes de zero entre os quantis da distribuição de rendimento. Logo, como tem parâmetros estimados significativamente diferentes de zero, os quantis analisados seriam estatisticamente diferentes, sugerindo a estimação do modelo pelo método da regressão quantílica.

Cabe destacar que, por meio deste teste, foi possível identificar os quantis que seriam utilizados para segmentar a amostra. Assim, chegou-se a conclusão de que os considerados estatisticamente diferentes são Q25, Q50 e Q75. 
Tabela 3 - Testes de igualdade interquantil para os trabalhadores da Região Norte do Brasil, 2013.

\begin{tabular}{|c|c|c|c|c|}
\hline Diferença interquantil & Entre ( & $\mathrm{Q} 50$ & Entre Q & e Q75 \\
\hline Variáveis & Teste $t$ & $\mathrm{P}>|\mathrm{t}|$ & Teste $t$ & $P>|t|$ \\
\hline E1 (4 a 7 anos) & 2,07 & 0,038 & $-0,33$ & 0,742 \\
\hline E2 (8 a 10 anos) & 2,28 & 0,022 & 0,63 & 0,531 \\
\hline E3 (11 anos) & 3,07 & 0,002 & 3,03 & 0,002 \\
\hline $\mathrm{E} 4(>11$ anos $)$ & 11,2 & 0,000 & 6,64 & 0,000 \\
\hline Idade & $-3,33$ & 0,178 & $-3,38$ & 0,000 \\
\hline Idade $^{2}$ & $-3,03$ & 0,761 & $-1,48$ & 0,020 \\
\hline Urbano & $-2,44$ & 0,015 & 0,96 & 0,335 \\
\hline Negros & 3,24 & 0,001 & 1,05 & 0,001 \\
\hline Pardos & $-0,79$ & 0,002 & $-2,40$ & 0,138 \\
\hline Construção & 0,43 & 0,001 & $-0,48$ & 0,295 \\
\hline Comércio & 1,51 & 0,432 & $-0,07$ & 0,016 \\
\hline Adm. pública & 0,3 & 0,665 & $-2,33$ & 0,633 \\
\hline Serviços & 2,14 & 0,130 & 4,71 & 0,943 \\
\hline Sexo & 1,35 & 0,033 & 4,34 & 0,000 \\
\hline Constante & 2,34 & 0,019 & $-0,35$ & 0,727 \\
\hline
\end{tabular}

Fonte: Resultados com base nos dados da PNAD 2013.

Além disso, foi realizado um teste de heterocedasticidade BreuschPagan/Cook-Weisberg, cuja hipótese nula do teste indica a homocedasticidade (Tabela 4). Desse modo, como o valor calculado da estatística foi 702,94, rejeitase a hipótese nula de erros homocedásticos no nível de 1\% e assume-se que existe a presença de heterocedasticidade. Portanto, houve a necessidade de estimar o modelo mantendo a suposição de erros independentes e relaxando as suposições de erros identicamente distribuídos. Isto é, semelhante à consideração de errospadrão robustos em regressões lineares. Com isso, geraram-se erros-padrão por meio do método de Bootstrap, garantindo a eficiência do modelo.

Ainda na Tabela 4, apresentam-se os resultados das equações mincerianas para os diferentes quantis de rendimentos. Assim, de acordo com a Teoria do Capital Humano, a variável escolaridade (proxy da produtividade do trabalhador) contribui diretamente e positivamente para a renda do trabalhador do Norte do Brasil (BECKER, 1964; MINCER, 1974; LANGONI, 1973; ARRAES; MARIANO, 2014; DALBERTO, 2014). E, se comparado nos três quantis, os trabalhadores que possuem 11 anos de estudo ou mais (ensino superior) com os trabalhadores 
que possuem 11 anos de estudo (ensino médio) e os trabalhadores que possuem de entre 8 anos a 10 anos de estudo (pelo menos Ensino Fundamental), percebese que o primeiro grupo apresenta maiores rendimentos, além de se considerar o impacto maior para os indivíduos que compõem o quantil 75.

Destaca-se que em nenhum dos três quantis analisados o nível de escolaridade dos trabalhadores que possuíam até 7 anos de estudos afetou significativamente o rendimento do trabalho. Isso mostra que mesmo para trabalhos com menor remunerariam, esse nível educacional já não seria capaz de gerar produtividade suficiente para afetar o rendimento do trabalhador, refletindo então a não significância da relação entre as variáveis. Este resultado é semelhante aos encontrados por Cirino e Cassuce (2012) e Dalberto (2014).

Intuitivamente, e aportado na Teoria do Capital Humano, esta relação positiva é explicada pela capacidade de investimento dos indivíduos em educação que produz efeitos em suas habilidades, logo, em sua produtividade marginal, fazendo com que haja ganhos em seus rendimentos (BECKER, 1964; MINCER, 1974). E isto pode estar intimamente ligado à relação entre atividades que auferem grande rendimento e profissionais altamente capacitados, ou seja, à medida que os indivíduos adquirem certo grau de conhecimento e habilidade, eles conseguem assumir atividades que auferem rendas maiores. Dessa forma, espera-se que, à medida que se aumenta os quantis, a influência da educação aumente.

Quanto à idade, ou seja, a proxy da experiência do trabalhador, foi percebida uma relação direta e positiva com o rendimento do indivíduo, também previsto pela teoria do capital humano, em todas as equações mincerianas, com maior impacto para os indivíduos que compõem o quantil 75 (BECKER, 1964; MINCER, 1974). A relação negativa entre a idade ao quadrado e a renda do indivíduo corrobora a hipótese de depreciação do capital humano previsto na teoria, ou seja, o impacto da idade sobre a remuneração dos trabalhadores é positiva, todavia, é decrescente até o ponto máximo da parábola, haja vista a relação entre estas duas variáveis. Após este ponto o impacto mencionado tornase negativo (BECKER, 1964; MINCER, 1974; DALBERTO, 2014). Este efeito parábola é observado em todos os quantis.

De acordo a Teoria do Capital Humano, a relação entre o efeito parábola e os rendimentos ocorre porque os investimentos em educação e treinamento no trabalho estão concentrados na juventude dos trabalhadores, e à medida que os trabalhadores envelhecem o desgaste físico e mental e o trade off entre trabalhar e investir em suas habilidades exige cada vez mais esforços para obter retornos em termos de remuneração, o que reflete em taxas decrescentes de renda ao longo de sua vida produtiva (BECKER, 1964; MINCER, 1974). Estes resultados são semelhantes aos encontrados por Mincer (1974), Kassouf (1998) e Dalberto (2014). 
Tabela 4 - Resultados da regressão quantílica para os trabalhadores da Região Norte do Brasil, 2013.

\begin{tabular}{|c|c|c|c|}
\hline $\begin{array}{c}\text { Teste para } \\
\text { heterocesdasticidade }\end{array}$ & $\begin{array}{l}\text { Estatística } \\
\left(\mathrm{chi}^{2}\right)\end{array}$ & Valor calculado & Prob $>$ chi $^{2}$ \\
\hline $\begin{array}{c}\mathrm{H}_{0:} \text { erros } \\
\text { homocesdásticos }\end{array}$ & $\begin{array}{c}\text { Breusch-Pagan / } \\
\text { Cook-Weisberg }\end{array}$ & 702,94 & 0.0000 \\
\hline Variáveis & Q25 & Q50 & Q75 \\
\hline \multirow[t]{2}{*}{ E1 (4 a 7 anos) } & $-0,1121$ & 0,0307 & 0,0231 \\
\hline & $(0,0169)$ & $(0,0264)$ & $(0,0325)$ \\
\hline \multirow[t]{2}{*}{ E2 (8 a 10 anos) } & $0,1322 *$ & $0,1734^{*}$ & $0,184^{*}$ \\
\hline & $(0,0183)$ & $(0,0250)$ & $(0,0294)$ \\
\hline \multirow[t]{2}{*}{ E3 (11 anos) } & $0,2945^{*}$ & $0,3434 *$ & $0,3985^{*}$ \\
\hline & $(0,0200)$ & $(0,2864)$ & $(0,0229)$ \\
\hline \multirow[t]{2}{*}{$\mathrm{E} 4(>11$ anos $)$} & $0,7458^{*}$ & $1,0130^{*}$ & $1,1935^{*}$ \\
\hline & $(0,0258)$ & $(0,0293)$ & $(0,0328)$ \\
\hline \multirow[t]{2}{*}{ Idade } & $0,0312^{*}$ & $0,0351 *$ & $0,0497 *$ \\
\hline & $(0,0030)$ & $(0,0051)$ & $(0,0062)$ \\
\hline \multirow[t]{2}{*}{ Idade $^{2}$} & $-0,0003^{*}$ & $-0,0002^{*}$ & $-0,0004^{*}$ \\
\hline & $(0,00004)$ & $(0,00007)$ & $(0,00009)$ \\
\hline \multirow[t]{2}{*}{ Urbano } & 0,1599* & $0,1182^{*}$ & $0,1335^{*}$ \\
\hline & $(0,0146)$ & $(0,0153)$ & $(0,0129)$ \\
\hline \multirow[t]{2}{*}{ Negros } & $-0,1041 *$ & $-0,1524 *$ & $-0,2178^{*}$ \\
\hline & $(0,0183)$ & $(0,0207)$ & $(0,0245)$ \\
\hline \multirow[t]{2}{*}{ Pardos } & $-0,0840^{*}$ & $-0,1194 *$ & $-0,1456^{*}$ \\
\hline & $(0,0097)$ & $(0,0117)$ & $(0,0149)$ \\
\hline \multirow[t]{2}{*}{ Construção } & $-0,3078^{*}$ & $-0,2298^{*}$ & $-0,2051 *$ \\
\hline & $(0,0241)$ & $(0,0223)$ & $(0,0335)$ \\
\hline \multirow[t]{2}{*}{ Comércio } & 0,0184 & 0,0038 & $-0,0495$ \\
\hline & $(0,0229)$ & $(0,0228)$ & $(0,0264)$ \\
\hline \multirow[t]{2}{*}{ Adm. pública } & $-0,0384$ & $-0,0308$ & $-0,0402$ \\
\hline & $(0,01688)$ & $(0,0150)$ & $(0,0243)$ \\
\hline \multirow[t]{2}{*}{ Serviços } & $0,0595^{*}$ & $0,0830 *$ & $0,0817^{*}$ \\
\hline & $(0,0164)$ & $(0,0187)$ & $(0,0249)$ \\
\hline \multirow[t]{2}{*}{ Sexo } & 0,2016* & $0,2245^{*}$ & $0,2920^{*}$ \\
\hline & $(0,0096)$ & $(0,0116)$ & $(0,0159)$ \\
\hline \multirow[t]{2}{*}{ Constante } & 0,2197* & $0,3672 *$ & $0,3470 *$ \\
\hline & $(0,0584)$ & $(0,0974)$ & $(0,0976)$ \\
\hline
\end{tabular}

Nota: desvio-padrão entre parênteses. * Significativo a 1\%; Desvio-padrão obtido por bootstrap com 500 repetições.

Fonte: Resultados com base nos dados da PNAD 2013. 
Ao comparar os efeitos marginais (Tabela 5), das variáveis de escolaridade entre os distintos quantis, verifica-se que os retornos para a escolaridade são maiores para o quantil 25, relativo à variável que indica 11 anos de estudo, ou seja, concluir o ensino médio tem maior impacto sobre o ganho daqueles que apresentam menores rendimentos. Isto não seria tão difícil de supor, uma vez que investimentos em capital humano para indivíduos com baixa renda num ambiente onde o mercado de trabalho está em constante transformação, pode ser altamente relevante para os ganhos salariais, enquanto que para as outras variáveis - E3 e E4 - não possuem tantas diferenças entre os quantis.

Outro aspecto interessante na Tabela 5 é que a variável que indica o efeito parábola, de acordo com Dalberto (2014), a depreciação do capital humano, apresenta efeitos mais intensos sobre os indivíduos que possuem remuneração mais baixa (Q25), ou seja, a idade média que demarca o ponto máximo com o rendimento é menor, se considerados os quantis 50 e 75 .

Quanto às variáveis relacionadas ao setor econômico, não é possível verificar nenhum tipo de tendência dos efeitos para os quantis da distribuição de rendimentos (Tabela 5). Na construção civil, observa-se que as diferenças se tornam cada vez maiores, na comparaçao com a indústria, quando se aproxima do $25^{\circ}$ quantil (Tabela 4). E em relação ao setor de serviços, a tendência é que a partir da mediana, a diferença entre este setor e a indústria aumente (Tabela 4).

$\mathrm{Na}$ Tabela 5 podemos perceber, ainda, que morar numa cidade localizada no Norte do Brasil surte maior efeito para aqueles que recebem rendimentos mais baixos (Q25), e depois para os indivíduos localizados na mediana. Esta informação pode reforçar a ideia de que o mercado de trabalho da cidade, devido ao nível maior de formalização, pode estar produzindo efeitos positivos sobre aqueles que recebem menos, visto que a formalização do mercado garante direitos trabalhistas, diferentemente do que ocorre na zona rural, onde a informalização do trabalho tende a ser mais intensa.

Quanto à variável cor, para a região Norte do Brasil pode-se perceber que os efeitos marginais (Tabela 5), sobre os ganhos são bem mais pertinentes quando a distribuição refere-se ao maior rendimento (Q75). Ou seja, os negros, além de receberem menos que brancos, e até mesmo pardos. Matos e Machado (2006) apontam que estes trabalhadores entram mais cedo no mercado de trabalho e tendem a deixar de lado os estudos, o que gera grande desigualdade educacional em comparação aos brancos, e o reflexo desta diferença de escolaridade (Tabela 2) é a ocupação de postos de trabalhos precários, que oferecem baixos salários.

Quanto à variável sexo, podemos observar que quanto mais próximo do quantil de maior rendimento (Q75), maior é o impacto sobre a renda dos 
indivíduos do gênero masculino (Tabela 4). Quanto aos efeitos marginais podem ser considerados oscilantes sobre a distribuição de rendimentos (Tabela 5).

Apesar de considerar a intensificação da participação das mulheres no mercado de trabalho ao longo deste século, sendo os fatores mais apontados em torno dessa maior participação a mudança de valores relativos ao papel social das mulheres (movimento feminista), a mudança de racionalidade das famílias no sentido de maximizar renda e, logo, o acesso aos bens de consumo, a elevação dos níveis de escolaridade das mulheres, a redução da taxa de fecundidade e do tamanho das famílias, estes dois últimos intimamente ligados à adoção de métodos anticonceptivos (ALVES; AMORIM; CUNHA, 1997; BRUSCHINI, 2000; MATOS; MACHADO, 2006).

No entanto, o acesso a esse mercado não garantiu igualdade de condições, mas a realização de uma discriminação salarial entre homens e mulheres, principalmente no que tange à ocupação dos mesmos postos de trabalho, onde as mulheres, que em geral são mais instruídas que os homens, recebem salários ou rendimentos menores que estes (ARAUJO; RIBEIRO, 2002; MATOS; MACHADO, 2006). Isto foi comprovado empiricamente nos dados das Tabelas 2 e 4.

Tabela 5 - Efeitos marginais da regressão quantílica para os trabalhadores da Região Norte do Brasil, 2013.

\begin{tabular}{lccc}
\hline & Q25 & Q50 & Q75 \\
\hline \multicolumn{1}{c}{ Valor do quantil } & $\mathbf{1 , 3 7}$ & $\mathbf{1 , 7 3}$ & $\mathbf{2 , 1 4}$ \\
\hline E1 (4 a 7 anos) & $-0,001$ & 0,0022 & 0,0013 \\
& $(0,0019)$ & $(0,0016)$ & $(0,0017)$ \\
E2 (8 a 10 anos) & $0,0208^{*}$ & $0,0217^{*}$ & $0,0185^{*}$ \\
& $(0,0031)$ & $(0,0026)$ & $(0,0027)$ \\
E3 (11 anos) & $0,085^{*}$ & $0,0787^{*}$ & $0,0735^{*}$ \\
& $(0,0056)$ & $(0,0047)$ & $(0,0049)$ \\
E4 ( $>$ 11 anos) & $0,1057^{*}$ & $0,1139^{*}$ & $0,1081^{*}$ \\
& $(0,003)$ & $(0,0025)$ & $(0,0026)$ \\
Idade & $0,7982^{*}$ & $0,7116^{*}$ & $0,8108^{*}$ \\
& $(0,0876)$ & $(0,0725)$ & $(0,0039)$ \\
Idade ${ }^{2}$ & $-0,2989^{*}$ & $-0,2279^{*}$ & $-0,2567^{*}$ \\
& $(0,0456)$ & $(0,0379)$ & $(0,0393)$ \\
Urbano $^{*}$ & $0,0969^{*}$ & $0,0568^{*}$ & $0,0517^{*}$ \\
& $(0,0087)$ & $(0,0073)$ & $(0,0074)$ \\
Negros $^{*}$ & $-0,0067^{*}$ & $-0,0078^{*}$ & $-0,009^{*}$ \\
& $(0,0011)$ & $(0,0009)$ & $(0,0098)$
\end{tabular}




\begin{tabular}{lccc} 
Pardos & $-0,0404^{*}$ & $-0,0456^{*}$ & $-0,0448^{*}$ \\
Construção & $(0,0051)$ & $(0,0043)$ & $(0,0044)$ \\
& $-0,0275^{*}$ & $-0,0163^{*}$ & $-0,0117^{*}$ \\
Comércio & $(0,0019)$ & $(0,0015)$ & $(0,0015)$ \\
& 0,0014 & 0,0002 & $-0,0024$ \\
Adm. pública & $(0,0015)$ & $(0,0012)$ & $(0,0013)$ \\
& $-0,0053$ & $-0,0033$ & $-0,0036$ \\
Serviços & $(0,0024)$ & $(0,0020)$ & $(0,0021)$ \\
& $0,0207^{*}$ & $0,0229^{*}$ & $0,0182^{*}$ \\
Sexo & $(0,0054)$ & $(0,0046)$ & $(0,0047)$ \\
& $0,0905^{*}$ & $0,08^{*}$ & $0,083)^{*}$ \\
Constante & $(0,0045)$ & $(0,0038)$ & $(0,0039)$ \\
& - & - & - \\
\hline
\end{tabular}

* Significativo a $1 \%$

Fonte: Resultados com base nos dados da PNAD 2013.

E por último, utilizando o coeficiente de determinação R1, que mede o relativo sucesso do modelo, foi gerado o Figura 1. A partir da análise do gráfico, pode-se verificar que a contribuição das variáveis de interesses, escolaridade do trabalhador e sua experiência advinda do trabalho tende a aumentar a taxas crescentes até o quantil 80 e posteriormente começa a cair, perdendo importância para o quantil 95 que representaria, proporcionalmente, elevadíssimos níveis de rendimento. Dessa forma, para trabalhadores com alto nível de rendimento, a escolaridade e a experiência teriam o mesmo poder de explicação da remuneração do que para trabalhadores que recebem baixa renda.

Esta tendência de certa forma valida a ideia de que investimentos na educação dos indivíduos geram retornos em termos de rendimentos mais elevados para os trabalhadores, conforme a Teoria do Capital. Desse modo, este resultado mostra que, além do aumento dos retornos, o ganho torna-se mais significativo nos estratos de renda mais alta (DALBERTO, 2014). 
Figura 1 - Coeficientes de determinação (R1) da regressão quantílica para os trabalhadores da Região Norte do Brasil, 2013.

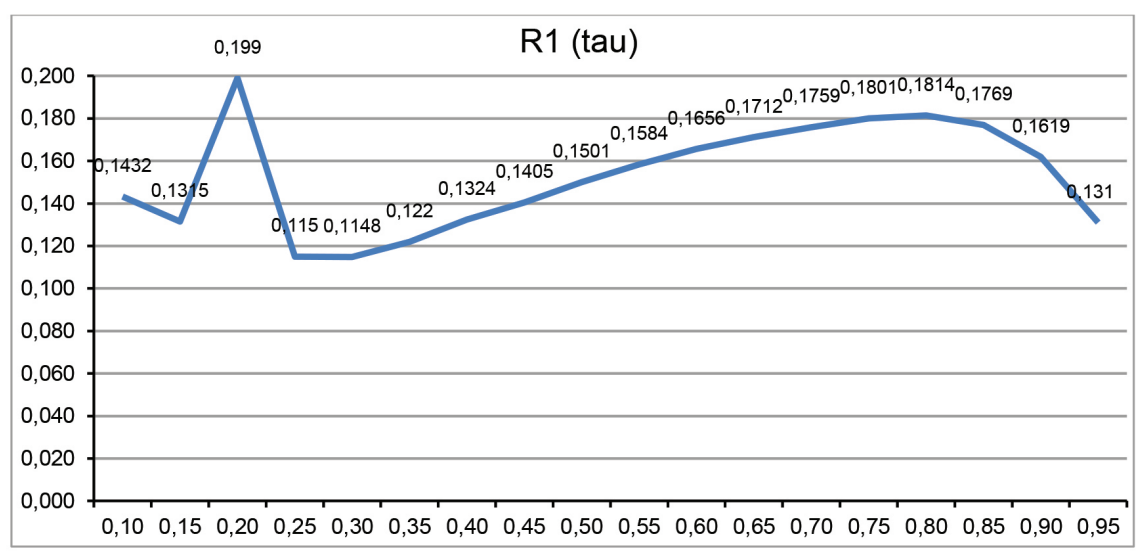

Fonte: Resultado com base nos dados da PNAD 2013

\section{CONSIDERAÇÕES FINAIS}

Conforme a teoria adotada aqui, a experiência do trabalhador e a sua escolaridade relacionam-se positivamente com os rendimentos deste trabalhador, com a ressalva da relação não linear entre a experiência e a renda.

A análise dos impactos ou de como a educação e a experiência relacionamse com a renda nos distintos estratos mostrou que os retornos são maiores à medida que os indivíduos apresentam maiores rendimentos. Além de verificar que indivíduos com ensino superior, seja completo ou não, apresentam maiores rendimentos, e que o impacto é maior para os indivíduos que compõem o quantil 75 , enquanto que a análise dos efeitos marginais apontou que concluir o ensino médio tem maior impacto sobre o ganho daqueles que apresentam menores rendimentos. Ou seja, além dos impactos destas variáveis serem positivos nos rendimentos dos trabalhadores, a intensidade sobre a magnitude também difere de acordo com os distintos quantis da amostra.

Assim como se verifica para o Brasil, o Norte também apresenta traços de discriminação por gênero e cor neste mercado de trabalho, o que pode refletir na precarização do trabalho para estes trabalhadores, onde atributos não econômicos e desvinculados da capacidade técnica e produtiva dos indivíduos norteiam as relações de salários neste mercado, contrariando todo o aporte teórico da abordagem, segundo os investimentos em capital humano. Isto ressalta que para usufruir das oportunidades geradas no mercado de trabalho, estes trabalhadores devem se qualificar constantemente, no sentido de inserir-se neste mercado pelo grau de conhecimento acumulado. No entanto, sugerem-se maiores estudos neste 
aspecto, a fim de melhor qualificar estes fatores e a dinâmica deste mercado.

Enfim, de forma agregada, ao abordar um tema relacionado à educação, principalmente de qualificação de mão de obra, busca-se discutir sobre as bases do desenvolvimento econômico e políticas capazes de alterar o nível de produto e, consequentemente, o nível de renda. E se considerarmos a renda como proxy ou medida de bem-estar para os trabalhadores, alterar este nível via investimento na educação desses indivíduos é primordial para aumentar a medida de bem-estar geral da economia, uma vez que representam a maioria.

\section{REFERÊNCIAS}

ALVES, E. L. G.; AMORIM, B. M. F.; CUNHA, C. H. M. Emprego e ocupação: algumas evidências da evolução do mercado de trabalho por gênero na Grande São Paulo - 1988/1995. IPEA, Texto para discussão, Rio de Janeiro, n. 497, jul. 1997.

ARAUJO, R. M. L.; OLIVEIRA, R.; CEA, G. S. A pesquisa em trabalho e educação nas regiões norte e nordeste. Trabalho Necessário, v. 13, n 20. 2015. Disponível em www.uff.br/trabalhonecessario. Acesso: 19/04/2016

ARAÚJO, V. F., RIBEIRO, E. P.Diferenciais de rendimentos por gênero no Brasil: uma análise regional.Revista Econômica do Nordeste, v. 33, n. 2, p. 196-217. 2002.

ARRAES, R.A.; MARIANO, F.Z. Endogeneidade da educação na previsão da taxa de retorno: avaliação metodológica e aplicação para regiões brasileiras e estados selecionados.Rev. Econ. NE, Fortaleza, v. 45, n. 2, p. 125-139, abril/jun., 2014.

BECKER, B. K. Revisão das políticas de ocupação da Amazônia: é possível identificar modelos para projetar cenários?ParceriasEstratégicas,MCT, n ${ }^{\circ} 12$, p. 135-159, 2001.

BECKER, G. S. Investiment in human capital: A theoretical analisys. Journalof Political Economy, v. 70, n. 5, p. 9-49, 1962.

BECKER, G. S. Human capital a theoretical and empirical analysis, with special reference to education. New York: Columbia University Press, 1964.

BERNDT, E.R. The practice of econometrics classic and contemporary. 8. ed. Boston,Massachusetts: Addison-Wesley, 1996.

BRUSCHINI, C. Gênero e trabalho no Brasil: novas conquistas ou persistência da discriminação? In: ROCHA, M. I. B. Trabalho e gênero: mudanças, permanências e desafios. São Paulo: Editora 34, 2000. P. 13-58.

CARDOSO JR, J. C. De Volta para o Futuro? Quão Sustentável Promete Ser a Recuperação Atual do Emprego Formal no Brasil? In: MONTAGNER, 
P.; PIRES, T.; VELHO, S. (org.). Análise da Pesquisa Nacional por Amostra de Domicílios - PNAD: 2005. Mercado de trabalho - Brasília: MTE, CGEE, AI, 2007. $193 \mathrm{p}$.

CIRINO, J. F.; CASSUCE, F. C. C. Geração de emprego e renda e a qualidade da educação no estado de Minas Gerais.Perspectiva Econômica (São Leopoldo. Online), v. 8, n. 2, Jul./Dez, p. 67-81, 2012.

CIRINO, J.F. Participação feminina e rendimento no mercado de trabalho: análises de decomposição para o Brasil e as regiões metropolitanas de Belo Horizonte eSalvador. 2008.188 p. Tese (Doutorado em Economia Aplicada) Universidade Federal de Viçosa, Viçosa, MG, 2008.

CUNHA, D. R.; VASCONCELOS, E. A. S.; PEREIRA, J. P. A.; MOREIRA, I. T. Mercado de Trabalho no Brasil. Características da Informalidade. Revista de Desenvolvimento Econômico. Ano XIII, n. 24. Dezembro/2011.

DALBERTO, C. R. Formalidade vs informalidade no mercado de trabalho brasileiro: uma investigação dos diferenciais de rendimento. 2014. Dissertação (Mestrado em Economia) - Universidade Federal de Viçosa, Viçosa, MG, 2014.

ESPERIDIAO, F;; GOMES, R. R. Retornos médios a educação nas regiões brasileiras: uma aplicação dos Microdados da Pnad para 2001 e 2011. In: XVIII Encontro de Economia da Região Sul (ANPEC SUL), 2015, Porto Alegre (RS). Encontro de Economia da Região Sul, 2015.

FERREIRA, R. S. A.; SOUZA, V. S. M.; HARB, A. G. Mercado de trabalho formal na região norte: uma análise por gênero. In: XXI SIMPOI (Simpósio de Administração da Produção, Logística e Operações Internacionais), 2013, São PauloSP. Anais do XXI SIMPOI da FGV, 2011. Disponível em http:/ /www.simpoi.fgrsp. br/arquivo/2011/artigos/E2011_T00238_PCN41727.pdf. Acessoem: 2014-10-19.

GAREN, J. The returns to schooling: A selectivity bias approach with a continuous choice variable.Econometrica, vol. 52, n.5, p. 1199-1218, 1984.

HECKMAN, J. J. Shadow prices, market wages and labor supply. Econometrica, 42(4):679-694.1974.

HECKMAN, J. J. Sample selection bias as a specification error. Econometrica, v. 47, n. 1, p. 153-161, jan. 1979.

KASSOUF, A. L. The wage rate estimation using the Heckman Procedure. Revista de Econometria, v. 14, n. 1, p. 89-107, abr./out.1994.

KASSOUF, A. L. Wage gender discrimination and segmentation in the Brazilian labour market. Economia Aplicada, v. 2, n. 2, p. 243-269, abr./jun.1998.

KOENKER, R.; BASSET, G. Regressionsquantiles.Econometrica, v. 1, n. 46, p. 33-50, 1978. 
LANGONI, C.G. Distribuição de renda e desenvolvimento econômico do Brasil. Rio de Janeiro: Expressão e Cultura, 1973.

IBGE. Notas metodológicas PNAD 2011. Rio de Janeiro: IBGE, 2011.

IBGE. Pesquisa Nacional por Amostra de Domicílios 2013. Rio de Janeiro, v. 33, 2013.

IBGE. Evidências baseadas em pesquisas domiciliares do ibge. Disponível em:<http:/ / docplayer.com.br/4799893-Evidencias-baseadas-em-pesquisasdomiciliares-do-ibge.html>. Acesso em: 02/2014.

MATOS, R. S.; MACHADO, A. F. Diferencial de rendimento por cor e sexo no Brasil (1987-2001).Econômica, v. 8, n. 1, p. 5-27, 2006.

MELLY, B. Estimation of counterfactual distributions using quantile regression. Reviewof Labor Economics, v. 68, p. 543-572, 2006.

MENDES, A. A. E. Desigualdades raciais e de gênero e a inclusão das mulheres negras na educação e no mercado de trabalho: 30 anos de história brasileira. Canoinhas: Universidade do Contestado Programa de Mestrado em Desenvolvimento Regional, 2013. p. 1-224. Disponível em: <www.unc.br/mestrado/ editais/Disserta_o_Andrea_Mendes_FINAL. pdf>. Acesso em: 19 abr. 2016.

MENEZES-FILHO, N. A. A. Evolução da educação no Brasil e seu impacto no mercado de trabalho. InstitutoFuturoBrasil, 2001.

MINCER, J. Schooling, experience, and earnings. New York: National Bureau of EconomicResearch: Columbia University, 1974.

REZENDE, M.; WYLLIE, R. Retornos para educação no Brasil: evidências empíricas adicionais. Economia Aplicada, v. 10, n. 3, p. 349-365, jul/set. 2006.

SACHSIDA, A.; LOUREIRO, P. R.A.; MENDONÇA, M. J.C. Um estudo sobre retornos em escolaridade no Brasil.Revista Brasileira de Economia, v. 58, n. 2, p. 249-265, abr./jun. 2004.

SANTOS, B. R. Modelos de regressão quantílica. Dissertação (Mestrado em Estatística) - Instituto de Matemática e Estatística, Universidade de São Paulo, São Paulo, 2012.Disponível em: <http://www.teses.usp.br/teses/disponiveis/45/45133/ tde-12042012-154333/>. Acesso em: 2014-10-19.

SILVA, D. L. G.; SANTOS, G. F.; FREGUGLIA, R. S. Distribuição espacial dos efeitos de aglomeração sobre os retornos à educação no brasil. Em: I Encontro de Economia Aplicada da Universidade Federal de Juiz de Fora, 2014.

SULIANO, D.C.; SIQUEIRA, M.L. Um estudo do retorno da educação na região nordeste: análise dos estados da Bahia, Ceará e Pernambuco a partir da recente queda da desigualdade. In: V Encontro de Economia do Ceará em Debate, Anais Eletrônicos... Fortaleza, IPECE, 2009. 